\title{
A SÍNDROME DE COMPARTIMENTO DO ABDOME
}

\author{
ABDOMINAL COMPARTMENT SYNDROME
}

José Ivan de Andrade

Docente do Departamento de Cirurgia, Ortopedia e Traumatologia da Faculdade de Medicina de Ribeirão Preto da Universidade de São Paulo.

CorResPondÊnCIA: Prof.Dr. José Ivan de Andrade. Rua: Bernardino de Campos, 1000 - CEP 14015-130 - Ribeirão Preto - SP; Telefone: (016) 625-9270; Fax (016) 610-2229; E-mail: jidandra@fmrp.usp.br

ANDRADE JI de. A síndrome de compartimento do abdome. Medicina, Ribeirão Preto, 31: 563-567, out./dez. 1998.

RESUMO: A síndrome de compartimento abdominal é definida como alterações progressivas das funções orgânicas, secundárias ao aumento da pressão intra-abdominal, difíceis ou impossíveis de serem tratadas até que o abdome seja descomprimido. Embora a fisiopatologia da síndrome esteja bem documentada clínica e experimentalmente, ainda persistem controvérsias e o diagnóstico e o reconhecimento da síndrome devem ser efetuados no âmbito das unidades de terapia intensiva.

O quadro clínico consiste de: abdome distendido e tenso, oligúria progressiva na vigência de débito cardíaco, apropriado e hipóxia, com aumento da pressão das vias aéreas. A descompressão cirúrgica do abdome é o único tratamento disponível e reverte prontamente o quadro.

UNITERMOS: Abdome. Traumatismos Abdominais. Insuficiência Renal. Anoxia.

\section{INTRODUÇÃO}

A síndrome de compartimento do abdome é uma entidade clínica na qual o aumento da pressão em um segmento anatômico, limitado, dificulta a circulação sanguínea, alterando a função e ameaçando a vitalidade dos tecidos. São sobejamente conhecidos os efeitos do aumento da pressão nos espaços limitados pelas fáscias nos membros superiores e inferiores e os benefícios advindos da descompressão pela fasciotomia. Por outro lado, os efeitos adversos do aumento da pressão intra-abdominal (PIA) têm merecido pouca atenção.

Entretanto, os efeitos deletérios do aumento da PIA já vêm sendo relatados de longa data. No limiar do século XX, Emerson ${ }^{(1)}$ demonstrou que pequenos animais de experimentação sucumbiam quando a PIA era abruptamente elevada de 27 para $46 \mathrm{~cm}$ de $\mathrm{H}_{2} \mathrm{O}$. Aparentemente, as mortes decorriam de insuficiência respiratória.
Thorington \& Schmidt ${ }^{(2)}$ observaram melhora da função renal após a execução da paracentese em um paciente com ascite por neoplasia. A partir desta constatação, desenvolveram um modelo experimental em cães, comprovando que uma elevação da PIA entre 15 e $30 \mathrm{~mm} \mathrm{Hg}$ resultava em oligúria e que aumentos superiores a $30 \mathrm{~mm} \mathrm{Hg}$ provocavam anúria.

$\mathrm{O}$ assunto caiu no ostracismo, até que, na década de 80 , Kron et al. ${ }^{(3)}$ e Richards et at. ${ }^{(4)}$ voltaram a demonstrar quadros de oligúria, em pacientes com elevação da PIA, geralmente associada à hemorragia pós-operatória.

\section{CONCEITO E ETIOLOGIA}

A síndrome de compartimento do abdome (SCA) é definida como um conjunto de disfunções orgânicas, decorrentes do aumento da PIA, prontamente revertidos pela descompressão da cavidade abdo- 
minal ${ }^{(5)}$. Clinicamente, observa-se um abdome distendido e tenso, aumento da pressão inspiratória, hipercapnia refratária à administração de oxigênio e oligúria. $\mathrm{O}$ aumento de volume de qualquer uma das estruturas abdominais pode resultar em elevação da PIA ${ }^{(6)}$. Elevações clinicamente significativas são mais comuns nas grandes hemorragias abdominais.

No trauma, vários fatores podem atuar simultâneamente, com adição de seus efeitos. Lesões extensas das vísceras parenquimatosas resultam num sangramento profuso e no choque hemorrágico. Traumas dos grandes vasos estão, usualmente, associados a hematomas retroperitoneais volumosos, que elevam a PIA $^{(7)}$. Ademais, muitas lesões extensas dos órgãos abdominais, em pacientes profundamente chocados, podem exigir o uso do "empacotamento" temporário das vísceras com compressas ${ }^{(8 / 11)}$. O edema das alças intestinais é comum na vítima de trauma e pode resultar da isquemia e reperfusão e da evisceração peroperatória prolongada. Esta leva ao edema pelo estiramento dos vasos sangüíneos e linfáticos do mesentério. Some-se a estes fatores, o edema do retroperitônio e a ascite aguda, conseqüentes à isquemia e reperfusão e à ressuscitação com grandes volumes de soluções cristalóides $^{(12)}$.

A SCA também vem sendo descrita nas seguintes situações: insuflação peritoneal durante cirurgia laparoscópica ${ }^{(13)}$, transplante hepático ${ }^{(14)}$, sangramento intra-abdominal, pós-operatório ${ }^{(15,16,17)}$, utilização da vestimenta pneumática antichoque ${ }^{(18,19)}$, complicações da cirurgia do aneurisma da aorta abdominal ${ }^{(15)}$, gran- des tumores abdominais ${ }^{(20)}$. A ascite também tem sido apontada como causa de $\mathrm{SCA}^{(21)}$. A Tabela I resume a etiologia da SCA.

\section{FISIOPATOLOGIA}

As principais alterações fisiopatológicas, relacionadas ao aumento da PIA, estão listadas na Tabela II.

\subsection{Sistema cardiovascular}

As respostas cardiovasculares no homem são muito semelhantes às observadas em animais de experimentação ${ }^{(14,22,23)}$. O aumento da PIA resulta em redução significativa do débito cardíaco ${ }^{(14,23 / 26)}$. O comprometimento do débito cardíaco deve-se a aumento da pressão intratorácica, aumento da resistência vascular, periférica e redução do retorno venoso. As alterações do débito cardíaco podem ser observadas mesmo nas situações em que a PIA é baixa (10-15 mm Hg) ${ }^{(24,26)}$. A redução do débito cardíaco pode ser revertida pela infusão de fluidos ${ }^{(12)}$.

O diafragma é empurrado cranialmente, pelo aumento da PIA, com conseqüente redução do volume e da complacência do tórax ${ }^{(14,27)}$. A compressão pulmonar resulta num aumento da pressão da via aérea $^{(6,12)}$ e da resistência vascular, pulmonar ${ }^{(28)}$, além de alterações da ventilação/perfusão ${ }^{(15)}$. A gasometria mostra hipoxemia e hipercarbia ${ }^{(25)}$. O aumento da resistência vascular periférica deve-se, provavelmente, à compressão mecânica do leito capilar ${ }^{(29)}$.

\footnotetext{
Tabela I - Fatores etiológicos relacionados à elevação da pressão intra-abdominal e que, potencialmente, podem resultar na síndrome de compartimento do abdome. Adaptado de Schein M et al. ${ }^{(6)}$

Aumento Agudo

Espontâneo

Peritonite, abscesso intra-abdominal, obstrução intestinal, íleo adinâmico, ruptura de aneurisma da aorta abdominal, trombose venosa mesentérica, pneumoperitônio, pancreatite aguda

- Pós-operatório

Peritonite pós-operatória, hemorragia intraperitoneal, abscesso intra-abdominal, íleo adinâmico, dilatação gástrica aguda

- Trauma

Edema visceral após tratamento do choque, hemorragia intraperitoneal, hemorragia retroperitoneal

- Latrogenia

Cirurgia vídeolaparoscópica, vestimenta pneumática, antichoque, correção de hérnias da parede abdominal ou diafragmáticas volumosas, "empacotamento" de lesões hemorrágicas com compressas, fechamento de laparotomia sob tensão

Aumento Crônico Grandes tumores intra-abdominais, ascite, diálise peritoneal, ambulatorial, gravidez
} 


\begin{tabular}{|c|c|c|c|}
\hline Parâmetro & Aumentado & Diminuído & Inalterado \\
\hline Pressão arterial, média & - & - & + \\
\hline Freqüência cardíaca & + & - & - \\
\hline $\begin{array}{l}\text { Pressão encunhada do capilar } \\
\text { pulmonar }\end{array}$ & + & - & - \\
\hline Pico de pressão da via aérea & + & - & - \\
\hline Pressão venosa, central & + & & \\
\hline Pressão pleural & + & - & - \\
\hline Pressão da veia cava inferior & + & - & - \\
\hline Pressão da veia renal & + & - & - \\
\hline Resistência vascular sistêmica & + & - & - \\
\hline Débito cardíaco & - & + & - \\
\hline Retorno venoso & - & + & - \\
\hline Fluxo sangüíneo visceral & - & + & - \\
\hline Fluxo sangüíneo renal & - & + & - \\
\hline Taxa de filtração glomerular & - & + & - \\
\hline Pressão intracraniana & + & - & - \\
\hline Pressão de perfusão cerebral & - & + & - \\
\hline $\begin{array}{l}\text { "Complacência" da parede } \\
\text { abdominal }\end{array}$ & - & + & - \\
\hline
\end{tabular}

houve redução do débito cardíaco, da perfusão renal e do débito urinário. Aos $40 \mathrm{~mm} \mathrm{Hg}$, os cães receberam fluidos por via intravenosa, com normalização do débito cardíaco, mas os parâmetros renais permaneceram inalterados. Eles apenas se normalizaram após a descompressão do abdome. Existem evidências de que a disfunção renal também é devida à compressão da artéria renal ${ }^{(34)}$, veia renal $^{(35)}$ e do próprio rim $^{(7)}$.

\subsection{Sistema nervoso central}

Uma publicação recente( ${ }^{(36)}$ demonstrou claramente os efeitos deletérios da elevação da PIA sobre o encéfalo. Um homem de trinta e três (33) anos sofreu queda e apresentava tórax flácido e pneumotórax, trauma cranioencefálico (múltiplas contusões focais, hemorragias parenquimatosas) e trauma abdominal (pequena laceração do lobo direito do fígado, à tomografia computadorizada) tratado conservadoramente. $\mathrm{O}$ paciente foi tratado por ventilação mecânica. No sexto pós-operatório, apresentou grande distensão abdominal, piora dos parâmetros gasométricos e hemodinâmicos, aumento da pressão intracraniana, queda da pressão de perfusão cerebral e aumento da drenagem de líquido cerebrospinhal. Nova tomografia com-

As anormalidades do retorno venoso podem ser creditadas à redução do fluxo sangüíneo, nas veias do retroperitônio e na cava inferior ${ }^{(6)}$.

A elevação da PIA reduz os fluxos da artéria hepática e veia porta, além da microcirculação hepática $^{(30)}$. Observa-se, também, redução da perfusão arterial do estômago, intestinos, pâncreas e baço ${ }^{(31,32)}$. O fluxo linfático pelo ducto torácico também diminui com o aumento da PIA ${ }^{(33)}$.

\subsection{Sistema urinário}

No homem, o aumento da PIA leva à insuficiência renal aguda, que melhora, prontamente, com a descompressão do abdominal ${ }^{(3,4,5,14,22)}$. Ocorrem queda do fluxo plasmático, renal e da taxa de filtração glome$\operatorname{rular}^{(7,17,25)}$. Em um estudo experimental ${ }^{(34)}$, foram monitorizados parâmetros hemodinâmicos e a função renal. Quando a PIA foi elevada de 0 a $40 \mathrm{~mm} \mathrm{Hg}$, putadorizada não mostrou alterações do encéfalo. O paciente foi submetido à laparotomia exploradora e o abdome mantido "aberto". Houve reversão imediata do quadro, com melhora do estado hemodinâmico e da respiração, declínio da pressão intracraniana e aumento da pressão de perfusão cerebral. O paciente recobrou a consciência oito dias após a laparotomia.

Os mecanismos pelos quais o aumento da PIA afeta a pressão intracraniana permanecem obscuros.

\subsection{Parede abdominal}

A pressão intra-abdominal depende da elasticidade e resistência da parede abdominal. A curva da relação volume/pressão (complacência) não é linear. À medida que a pressão intracavitária se eleva, a resistência da parede aumenta. Após um determinado ponto, pequenas elevações de volume aumentam desproporcionalmente à pressão. 


\subsection{Gradação da resposta}

As alterações fisiopatológicas manifestam-se gradativamente, à medida que a PIA se eleva. Existe uma classificação que relaciona a gravidade da SCA e os valores da PIA (Tabela III).

\begin{tabular}{|cc|}
\hline $\begin{array}{c}\text { Tabela III - Escore de gravidade na síndrome de } \\
\text { compartimento do abdome } \\
\text { Adaptado de Burch JM et al. }{ }^{(12)}\end{array}$ \\
\hline Escore & Pressão vesical $\left(\mathrm{cm} \mathrm{H}_{2} \mathrm{O}\right)$ \\
\hline I & $10-15$ \\
II & $15-25$ \\
III & $25-35$ \\
IV & $>35$ \\
\hline
\end{tabular}

\section{DIAGNÓSTICO}

O diagnóstico é simples e baseia-se nos seguintes achados: abdome tenso e distendido, oligúria progressiva com hidratação normal e hipóxia, com aumento da pressão da via aérea. A medida da pressão vesical confirma o diagnóstico. A bexiga urinária é um reservatório passivo, quando o seu conteúdo é inferior a $100 \mathrm{ml}$. Portanto, nestas condições, um cateter inserido em seu interior é capaz de aferir a PIA, sem o acréscimo da pressão gerada pelas contrações das fibras do detrusor.

A técnica de mensuração consiste de: 1) introduzir cateter de Foley e esvaziar a bexiga; 2) clampar a via de drenagem do cateter; 3) puncionar a via de aspiração com agulha calibre 18 e instilar cinco $\mathrm{ml}$ de salina; 4) conectar a seringa a um manômetro de água; 5) com o nível zero do manômetro fixado ao nível da sínfise púbica, proceder à leitura da pressão.

\section{TRATAMENTO}

A descompressão cirúrgica do abdome é o único tratamento para a $\mathrm{SCA}^{(12)}$. Durante a operação, pode ocorrer hipotensão arterial, transitória, iniciada a partir da abertura da cavidade abdominal ${ }^{(10)}$. Para prevenir descompensação circulatória durante a cirurgia, deve-se restaurar o volume circulante, maximizar a oferta de $\mathrm{O}_{2}$ e corrigir os distúrbios de coagulação e a hipotermia porventura existentes.

$\mathrm{Na}$ profilaxia dos possíveis efeitos da absorção de metabólitos acumulados durante a isquemia, é recomendável incluir, na hidratação, bicarbonato de sódio e manitol ${ }^{(10)}$. A assistolia, imediatamente após a descompressão foi descrita em quatro casos, sendo três fatais ${ }^{(10)}$. Por isto, é preferível, nos pacientes sem sinais de sangramento, realizar a descompressão do abdome dentro da própria unidade de tratamento intensivo, com o paciente monitorizado.

O abdome deve permanecer aberto até a reversão do quadro. Existem diferentes modalidades técnicas para proteção da parede e dos órgãos abdominais, cuja discussão foge do escopo do presente artigo.

ANDRADE JI de. Abdominal compartment syndrome. Medicina, Ribeirão Preto, 31: 563-567, oct./dec. 1998.

ABSTRACT: The abdominal compartment syndrome (ACS) is defined as an impairment of organ functions due to an increased intra-abdominal pressure (IAP). It becomes progressively difficult or impossible to deal with this impairment, unless IAP is reduced. Although the physiopathology of ACS has been well defined clinically and experimentally, much controversy still exists. This condition should be recognized in intensive care units. There are significant changes in hemodynamic, respiratory, renal, and metabolic functions.

The clinical findings include a tightly distended abdomen, progressive oliguria in spite of adequate cardiac output, and persistent hypoxia with increased airway pressure. Surgical abdominal decompression is the only treatment with improvement of the patient's cardiac, pulmonary, and renal function.

UNITERMS: Abdomen. Abdominal Injuries. Kidney Failure. Anoxia. 


\section{REFERÊNCIAS BIBLIOGRÁFICAS}

1 - EMERSON H. Intra-abdominal pressures. Arch Intern Med 7: 754-784, 1911.

2 - THORINGTON JM \& SCHMIDT CF. A study of urinary output and blood-pressure changes resulting in experimental ascitis. Am J Med Sci 165: 880-886, 1923.

3 - KRON IL, HARMAN PK \& NOLAN SP. The measurement of intra-abdominal pressure as a criterion for abdominal re-exploration. Ann Surg 199: 28-30, 1984.

4 - RICHARDS WO et al. Acute renal failure associated with increased intra-abdominal pressure. Ann Surg 197: 183-187, 1993.

5 - BENDAHAN $\mathbf{J}$ et al. Abdominal compartment syndrome. $\mathbf{J}$ Trauma 38: 152-153, 1995

6 - SCHEIN M et al. The abdominal compartment syndrome: the physiological and clinical consequences of elevated intra-abdominal pressure. J Am Coll Surg 180: 745-753,1995.

7 - JACQUES T \& LEE R. Improvement of renal function after relief of raised intra-abdominal pressure due to traumatic retroperitoneal hematoma. Anaesth Intensive Care 16: 478-482, 1988.

8 - HIRSHBERG A \& MATTOX K. "Damage control" in trauma surgery. Br J Surg 80: 1501-1502, 1993.

9 - ANDRADE JI et al. Qual é a melhor abordagem terapêutica nas hemorragias intra-abdominais catastróficas maciças? In: CASTRO LP; SAVASSI-ROCHA PR \& CARVALHO EB, ed. Tópicos em gastroenterologia 6. Medsi, Rio de Janeiro, p. 287-293, 1996.

10 - MORRIS $\mathrm{J}$ et al. The staged celiotomy for trauma. Issues in unpacking and reconstruction. Ann Surg 217: 576-586, 1993.

11 - SHARP KW \& LOCICERO RJ. Abdominal packing for surgically uncontrollable hemorrhage. Ann Surg 215: 467-475, 1992.

12 - BURCH JM et al. The abdominal compartment syndrome. Surg Clin North Am 76: 833-842, 1996.

13 - SAFRAN DS \& ORLANDO R. Physiologic effects of pneumoperitoneum. Am J Surg 167: 281-286, 1994.

14 - CULLEN DJ et al. Cardiovascular, pulmonary, and renal effects of massively increased intra-abdominal pressure in critically ill patients. Crit Care Med 17: 118-121, 1989.

15 - FIETSAM R et al. Intra-abdominal compartment syndrome as a complication of ruptured abdominal aortic aneurysm repair. Am Surg 56: 396-402, 1989.

16 - SHELLY MP et al. Haemodynamic effects following surgical release of increased intra-abdominal pressure. $\mathbf{B r} \mathbf{J}$ Anaesth 59: 800-805, 1987.

17 - PLATELL C, HALL J \& DOBB G. Impaired renal function due to raised intra-abdominal pressure. Intensive Care Med 16: 328-329, 1990.

18 - O'LEARY MJ \& PARK GR. Acute renal failure in association with a pneumatic antishock garment and with tense ascitis. Anaesthesia 46: 326-327, 1991.
19 - MCSWAIN NE. Pneumatic antishock garment: state of the art 1988. Ann Emerg Med 17: 506-525, 1988.

20 - CELORIA G et al. Oliguria from high intra-abdominal pressure secondary to ovarian mass. Crit Care Med 15: 78-79, 1987.

21 - SAVINO JA et al. Manipulation of ascite pressure in cirrhotics to optimize hemodynamic and renal function. Ann Surg 208: 504-509, 1988.

22 - DALY RC, MUCHA P \& FARRELL MB. Abdominal reexploration for increased intra-abdominal pressure and acute oliguric renal failure. Contemp Surg 35: 11-18, 1989.

23 - MELDRUM DR et al. Cardiopulmonary compromise following perihepatic packing of major liver injuries. Am J Surg 170: 537-542, 1995.

24 - WESTERBAND A et al. Cardiovascular changes during laparoscopic cholecystectomy. Surg Gynecol Obstet 175: 535-538, 1992.

25 - BARNES GE et al. Cardiovascular responses to elevation of intra-abdominal hydrostatic pressure. Am J Physiol 248: R209-R213, 1985.

26 - HO HS; GUNTHER RA \& WOLFE BM. Intraperitoneal carbon dioxide insufflation and cardiopulmonary functions. Arch Surg 127: 928-933, 1992

27 - IVANKOVICH AD et al. Cardiovascular effects of intraperitoneal insufflation with carbon dioxide and nitrous oxide in the dog. Anesthesiology 42: 281-287, 1975.

28 - IBERTI TJ et al. A simple technique to accurately determine intra-abdominal pressure. Crit Care Med 15: 1140-1142, 1987.

29 - LUCA A et al. Hemodynamic effects of acute changes in intra-abdominal pressures in patients with cirrhosis. Gastroenterology 104: 222-227, 1993.

30 - DIEBEL LN et al. Effect of increased intra-abdominal pressure on hepatic arterial, portal venous, and hepatic microcirculartory blood flow. J Trauma 33: 279-283, 1992.

31 - CALDWELL CB \& RICOTTA JJ. Changes in visceral blood flow with elevated intra-abdominal pressure. J Surg Res 43: 14-20, 1987.

32 - JAFFE V \& RUSSEL RCG. Fatal intestinal ischemia following laparoscopic cholecystectomy. Br J Surg 81: 1927-1928, 1994.

33 - WHITE CL. Manipulation of ascitic fluid in cirrhotics to optimize hemodynamic and renal function. Ann Surg 208: 504-511, 1988.

34 - HARMAN PK et al. Elevated intra-abdominal pressure and renal function. Ann Surg 196: 594-597, 1982.

35 - SHENASKI JH \& GILLENWATER JY. The renal hemodynamic and functional effects of external counter pressure. Surg Gynecol Obstet 134: 253-258, 1972.

36 - BLOONFIELD GL et al. Treatment of increasing intracranial pressure secondary to the acute abdominal compartment syndrome in a patient with combined abdominal and head trauma. J Trauma 39: 1168-1170, 1995.

Recebido para publicação em 04/03/98

Aprovado para publicação em 07/10/98 